\title{
Exploration and Cooperation Robotics on the Moon
}

\author{
Mohammad Alfraheed ${ }^{1}$, Abdullah O. Al-Zaghameem ${ }^{2}$ \\ ${ }^{1}$ Department of Mathematics and Computer Science, Tafila Technical University, Tafila, Jordan; ${ }^{2}$ Department of Mathematics \\ and Computer Science, Tafila Technical University, Tafila, Jordan. \\ Email: Alfraheed@ttu.edu.jo,aoz_0202@yahoo.com
}

Received June $17^{\text {th }}, 2013$; revised July $15^{\text {th }}, 2013$; accepted July $23^{\text {rd }}, 2013$

Copyright (C) 2013 Mohammad Alfraheed, Abdullah O. Al-Zaghameem. This is an open access article distributed under the Creative Commons Attribution License, which permits unrestricted use, distribution, and reproduction in any medium, provided the original work is properly cited.

\begin{abstract}
Space robotics are the development of general purpose machines that is capable of surviving (for a time, at least) in the rigors of the space environment, and performing exploration, assembly, construction, maintenance, servicing. Space Robots can perform tasks less expensively or on an accelerated schedule, with less risk and occasionally with improved performance while humans doing the same tasks. The moon is the natural next step in the exploration of our own universe. Understanding moon better will help us understand our neighbors in the solar system. In this paper, a concept of exploration and cooperation robotics on the moon is discussed. The concept requires not only to extend the exploration mission on the moon surface but also to address a way to integrate the developed robotics with each other. Sharing the information between robots is one of a concept's features to reduce lime and power consumption in the exploration process. Moreover, several challenges are discussed here, which prevent the concept from developing in outer space or on moon.
\end{abstract}

Keywords: Space Robotics; Exploration Robotics; Human-Robot Cooperation; Wheel Slippage of Planetary Robots

\section{Introduction}

Space robotics and other general purpose machines are developed that are capable of surviving in the rigors of the space environment and can perform special tasks (e.g. exploration, assembly, construction, maintenance, servicing etc.) [1]. Space robots are important to our overall ability to operate in space because firstly, they can perform tasks less expensively, with accelerated schedule, with less risk and with improved performance. Secondly, they can operate for long durations and stay in the stand by for long periods while traveling from the earth to the destination. Thirdly, they can enter dangerous environments which humans cannot access [1].

Since 1980s many ideas have been proposed to assemble space structures by autonomous free-flying robots in orbit; for example for the servicing missions to existing satellites [2]. However, the International Space Station (ISS) has not been constructed by such an autonomous-flying robot. Instead a Shuttle Remote Manipulator System (Canadarm 1) [3] and the Space Station Remote Manipulator System (Canadarm 2) [4] were used to assist the astronauts to construct the ISS.

In contrast, orbital free-flying space robots were used to retrieve and dock with existing satellites in orbit and then conduct the servicing tasks (e.g. replacing components, resupplying expendables, repairing, rescuing, reorbiting the satellite etc.) [5]. An engineering test satellite launched in November 1997 by JAXA japan Aerospace Exploration Agency is here mentioned as an example for free-flying robot [2]. Another example is Orbital Express, which was launched in March 2007, and was developed by DARPA (Defense Advanced Research Projects Agency) program. Figure 1 shows The DARPA Orbital Express spacecraft in orbit [6]. The Autonomous Space Transport Robotic Operations (ASTRO) servicing spacecraft is on the left of the figure, and approaching the NextSat, a surrogate next generation serviceable satellite, and preparing to rendezvous and dock.

The paper is organized as follows; a brief description for the exploration of planetary surfaces is given in Section 2. Section 3 describes the concept between a human and a robot in terms of space environment. In Section 4, the concept of the exploration and cooperation on the moon is discussed. Challenges preventing the concept from developing are discussed (Section 5). Finally, conclusion and future work are given in the last section. 


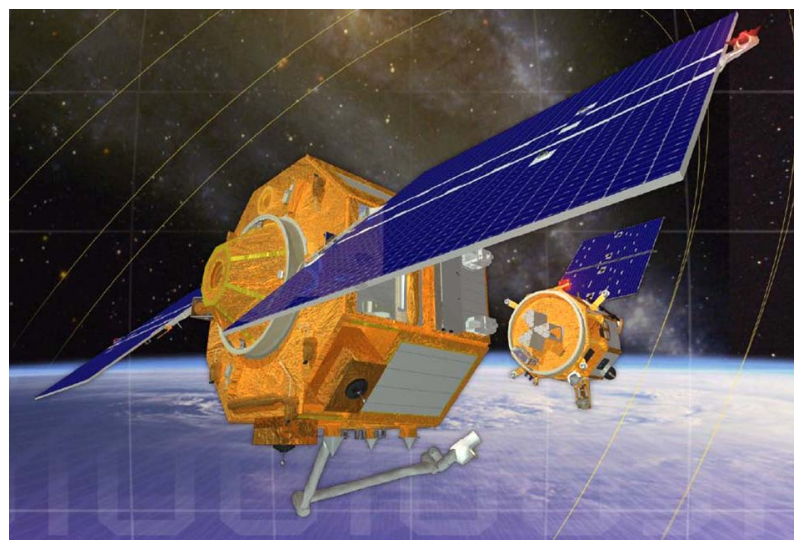

Figure 1. The DARPA orbital express spacecraft in orbit.

\section{Exploration of Planetary Surfaces}

For the exploration of planetary surfaces, robots have been contributing to expand the frontier of scientific knowledge and human access. In exploration missions, robots are required not only to collect soil samples but also to conduct in-situation analysis. Since robots consist of sensitive sensors, the landing process needs to be carried out carefully [2]. Recently, several mechanisms have been developed and released to land robots on planetary surface.

Asteroids and comets are referred to as minor planetary bodies. These asteroids and comets are also attractive destinations for robotic probes and scientific discoveries [7]. One of the tasks associated with asteroid robotics is to conduct a flyby observation of the asteroid to unveil the shape of its nucleus and to observe its orbital [2]. Hayabusa and Rosetta are two examples for robotics probes. The latter was developed by the European Space Agency and was launched in 2004. It consists of two elements the Rosetta space probe (shown in Figure 2) and the philae lander [8]. The philae lander is scheduled to land on the surface of the nucleus in 2014. To prevent the lander from bouncing off, two harpoons will be fired into the surface of the nucleus and additional drills are used to secure the lander. This allows an in-situation analysis of the nucleus [2].

\section{Cooperation between Humans and Robots}

The cooperation concept between a human and a robot is not a new concept. It has already been introduced and developed in many fields (e.g. industry). Recently, the Space environment has received the researcher's attention to develop that concept further to be robust against the harsh environment in the outer space. The robot can carry out many tasks for the astronauts such as intravehicular activities [9]. In case the astronaut needs immediately necessary information, the robot provides it much faster than the human because its reaction time is

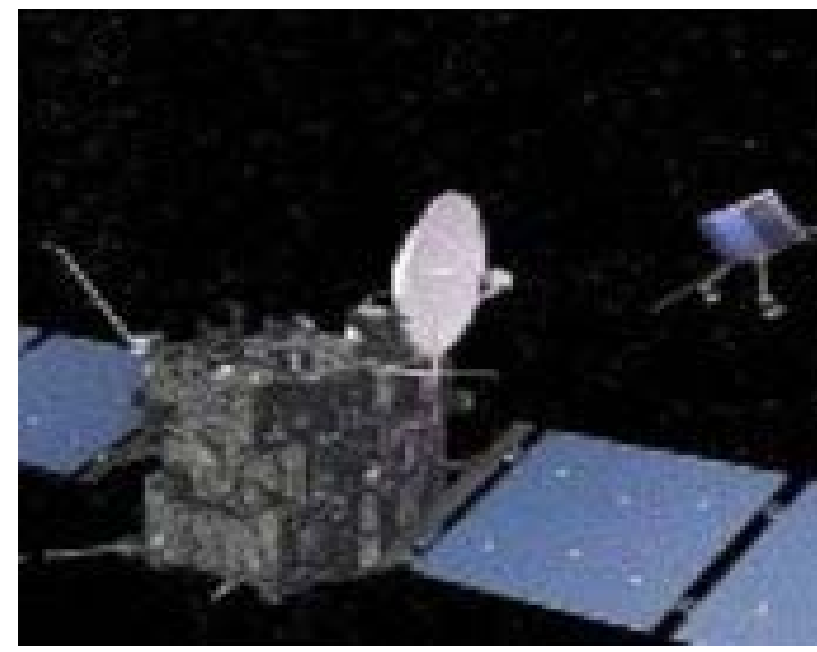

Figure 2. The Rosetta spacecraft.

shorter. Also, the robot can carry out tasks that require dexterity and strength. In addition, there is a percent of error if the human perform these tasks (i.e. extra-vehicular activities) [10].

The moon is the natural next step in the exploration of our own solar system [11,12]. Firstly because, a better understanding of the moon will help us to better understand our neighbor planets. Secondly, the human has learned much about the moon from the Apollo program. But now, the return to the moon is required for intensive Study. In other words, we need to go back to the moon so that we can live there for longer periods and work on the moon. Thirdly, research showed that the generation of electricity in vacuum is much more efficient than on earth is. So the moon's surface can be a good base to generate electricity with a highly efficient solar energy system. Moreover, moon dust contains all the materials needed to create solar cells, they need only to be collected and processed. The electricity is then transported to the earth via microwave signals [12].

In context of artificial intelligence and robotics, the most recent trend in the NASA is to emphasis on human-robotic cooperation as a part of plans to return to the moon [10].

JAXA will release an engineering experiment of the Astrobot (astronaut + robot) for moon and planetary exploration [11]. The European Space Agency (ESA) developed a robot, which is remotely operated by a human (shown in Figure 3). The next step for the Eurobot system is the exploration step in which the system will run autonomously based on the astronaut-assigned job descriptions. The European Space Agency (ESA) developed a robot, which is remotely operated by a human. The next step for the Eurobot system is the exploration step in which the system will run autonomously based on the astronaut-assigned job descriptions [13]. 


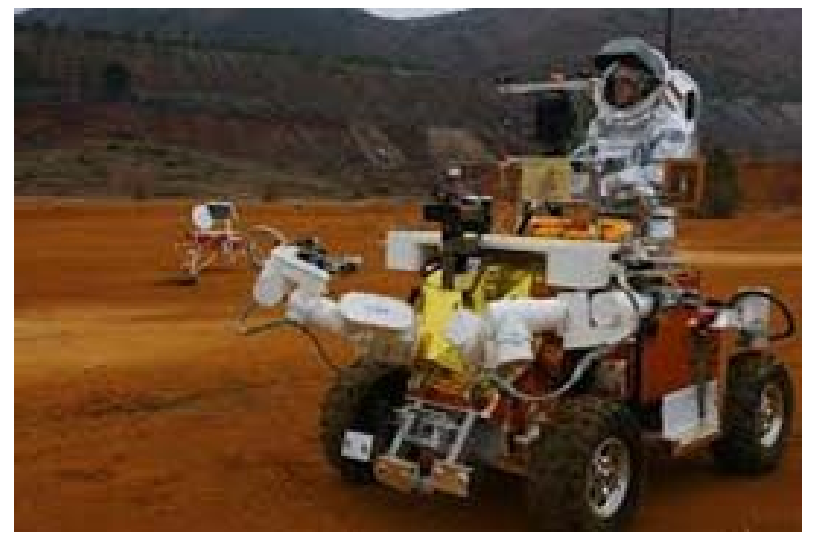

Figure 3. Astronaut at the controls of the eurobot ground prototype.

\section{Future Vision: Building a Space Station on the Moon}

To build a space station on the moon, several questions are highlighted: How will the space agency build its own base on the moon? Where should a moon base be constructed? And how can the current developed space robots assist astronauts to carry out the extra-vehicular activities with limited resources (time, power)? As an initial answer for these questions, a concept for exploration and cooperation robotics on the moon is introduced. Figure 4 shows an illustration of the concept for the exploration and cooperation robotics on the moon. The concept requires not only to extend the exploration mission on the moon surface but also to address a way to integrerate the developed robotics with each other.
In Figure 4, NASA's MARS Exploration Rover MER [14,15], the Robonaut 2 [10] and the Mars rover Curiosity [16] explore the Moon's surface together. Sharing the information between robots is one of a concept's features to reduce lime and power consumption in the exploration process. Each robot can send and receive the topographical maps generated to avoid an exploration of an already examined area and to ensure that the robots only travel on paths that were validated as safe. This information can also be used to determine a suitable construction area for the moon base-Rescuing and maintenance are assigned to a humanoid robot which also acts as a server to the space station and to connect the human operator in the space agency.

In more details for the concept (shown in Figure 5), the Humanoid Robot [17] is working a server robot which can run several tasks; Maintence, Rescuer in case the wheel slippage through pushing out the robot and Communication with the Space Station.

Date processing; within the concept, the server Robot will be responsible to receive data from the rover robot. For instance, the rover robot sends its generated 3D map generated by its sensors. The 3D map is integrated with other stored map. The updated version of the $3 \mathrm{D}$ stored map is then sent to the rover robot in order to expand its vision about the surroundings.

Moreover, the Server Robot is responsible to send the safety path to the rover instead of calculating it by the rover itself. In case the rover changes its path to dangerous area, an alert is sent to the rover by the server robot.

The rover robot has to carry out, in turn, the explora-

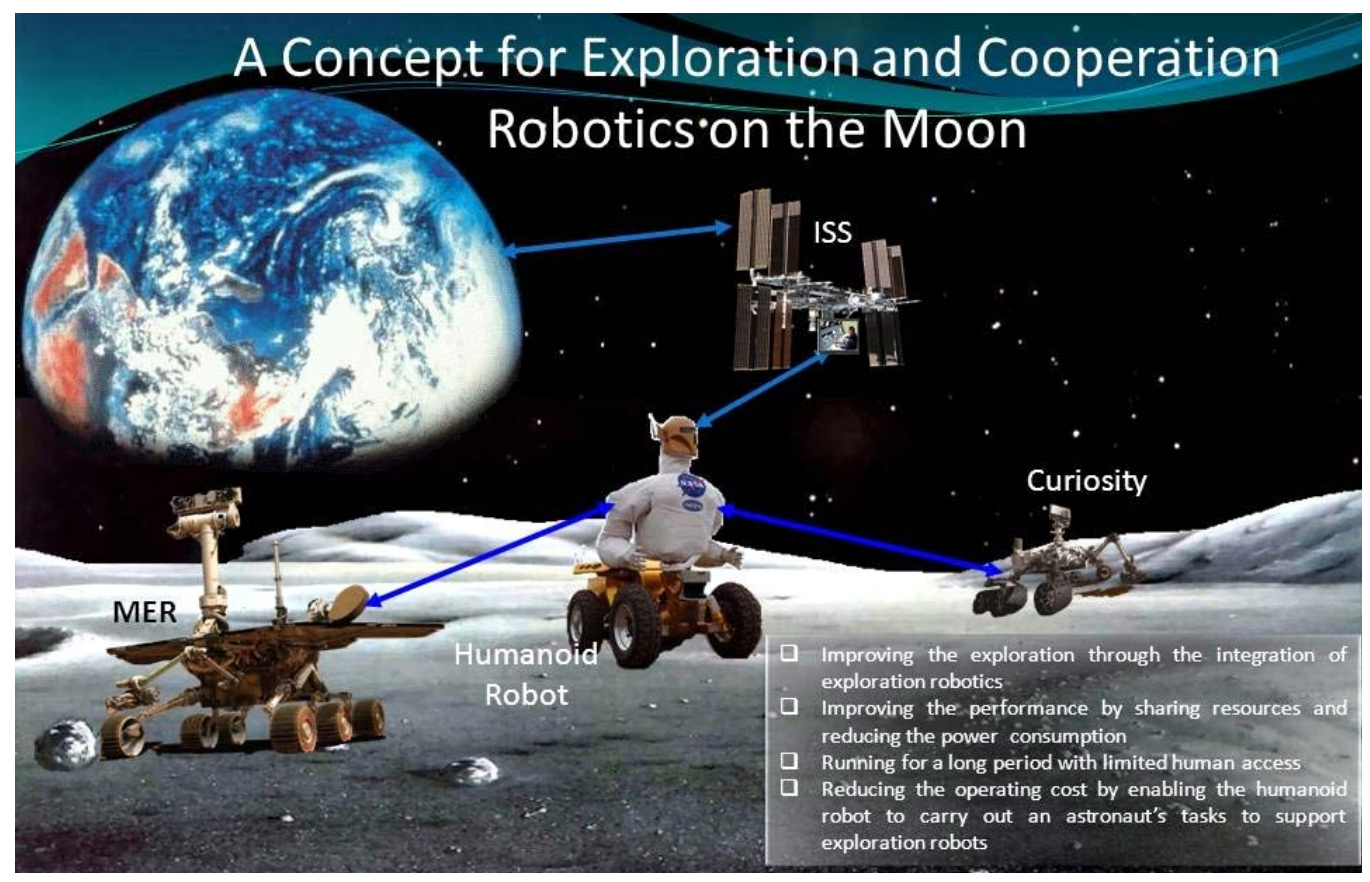

Figure 4. An illustration of the concept for exploration and cooperation robotics on the moon. 


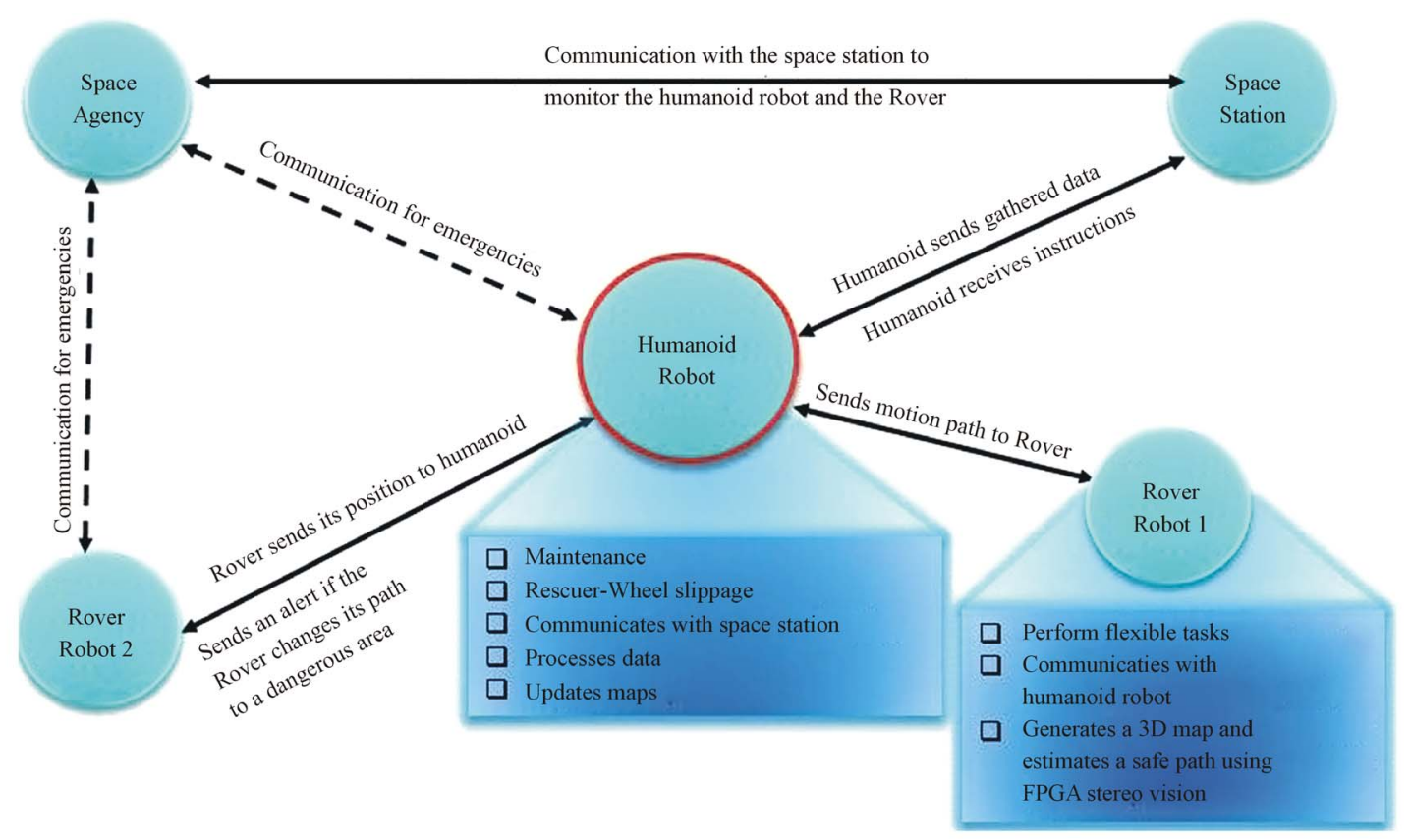

Figure 5. A detailed illustration of the concept for exploration and cooperation on the moon.

tion task. Within this concept, the exploration task is not fixed and it changed based on the description sent from the server robot. The server robot, in turn, receives the task via the communication established with astronaut in the space station.

\section{Challenges for Future Developments}

The cooperation concept between several of robots is not a new concept. It has been developed been developed before in many fields (i.e. industry, military applications). Due to several challenges, the concept introduced here cannot be developed in outer space especially, on the moon surface. These challenges are as follows:

\subsection{Challenge 1: Limited Resources and the High Degree of Autonomy Required for Planetary Robots}

The odometry measurements and mapping process [2] consume so much time and computation resources to be implemented [11]. In other words, Mapping and odometry processes are generally the most computationally expensive processes in autonomy navigation architectures, which is also required with a high accuracy to avoid obstacles. In both processes, the robot has to first stop and acquire data from its sensors. Based on this data, an evaluation has to be carried out for surroundings. An estimation process of the relative change in robot's pose has to be then computed. In the last step, mobility has to be achieved. As an example, MER (Spirit and Opportunity) [18] are powered by a $20 \mathrm{MHz}$ RAD6000 computer providing approximately 22 MIPS. Curiosity [16] is utilized by a newer RAD750 capable of operating at 200 $\mathrm{MHz}$ and $400 \mathrm{MIPS}$. Due to the limited resources to generate energy on other planets (i.e. Mars), the level of performance has to be restricted to these resources. As a solution proposed here are:

- Sharing and storing the generated 3D maps space environment between the robots.

- Reducing the running time to perform odometry processes through the use of the SVS from the FieldProgrammable Gate Array circuit (FPGA) [19,20].

\subsection{Challenge 2: Wheel Slippage of Planetary Robots}

The second challenge is the high degree of autonomy is required to avoid obstacles in the surface exploration. In practice, a low percentage of error guides the rover to the dangerous path. For instance, the planetary vehicles that travel sand dune-like terrain may encounter wheel slippage and loose traction control [21]. In late April 2005, Opportunity got stuck in a soft sand dune and due to significant wheel slippage; it took many weeks until it finally got back onto firm ground in early June 2005 [2]. As solutions proposed here are as follows:

- Technically, a terrestrial analog had been used to measure the amount of slippage of the drive wheels during a traversal of Mars-like terrain [22,23]. This measurement depends on the markings lane generated by the rover itself. So it would be suggested to develop an approach which is able to estimate the next area of ground if it is soft or not, and to sense the ex- 
cessive wheel sinkge. Therefore, the control system will be able to execute the suitable order for the rover before the vehicle becomes immobile.

- To check the next surface for the rover robot, it would be proposed to use the Stereo Odometry Vision which is equipped by FPGA circuit [20]. Since the latter has internal process and memory, it is able to carry out some computational tasks. So, the consumed energy is reduced

\subsection{Challenge 3: Safe Operation of the Humanoid Robot in Outer Space}

In the proposed concept, the humanoid robot plays an important role as server. So it has to meet the safe requirements to work in the space. These requirements are as follows:

- Fault Containment Regions to prove safety in case of an unexpected fault.

- Verification of the behavioral properties (reliability and robustness) of robots.

- A full set of teleportation gear for robots [24,25].

- Increase in bandwidth for the signal transmission to enhance the communication with the robot.

\section{Conclusion and Future Work}

Using robotics expands the frontier of scientific knowledge of space and humans can access to it, especially for planetary exploration. Moreover, Cooperation between humans and robots and cooperation between robots in space enable astronauts to carry out their tasks with lower risk and with improved performance. The next scientific step of humans is the return to the moon because a detailed knowledge of the moon enables us to improve our understanding of our solar system. In the context of the return to the moon, a concept of exploration and cooperation is discussed in the paper. In addition, challenges which prevent the concept from developing in outer space (on moon surface) are here discussed. Solutions are highlighted to overcome those challenges. One of these solutions is a vision-based approach. The latter is required to develop the visual odometry techniques in order to estimate the soft ground region with limited computational resources. Furthermore, the safe requirements for the humanoid robot are highlighted.

\section{REFERENCES}

[1] B. Wilcox, R. Ambrose and V. Kumar, "Chapter 3Space Robotics,” 2006. http://www.wtec.org/robotics/report/03-Space.pdf

[2] K. Yoshida, "Achievements in Space Robotics," IEEE Robotics \& Automation Magazine, Vol. 16, No. 4, 2009, pp. 20-28. doi:10.1109/MRA.2009.934818
[3] G. Bones and C. Nerves, "Canadarm—The Background,” 2013.

http://www.ieee.ca/millennium/canadarm/canadarm_back ground.html

[4] T. Tran, "NASA-Canadarm 2 and the Mobile Servicing System,” 2013.

http://www.nasa.gov/mission_pages/station/structure/ele ments/mss.html

[5] H. Nakanishi and K. Yoshida, "Impedance Control for Free-Flying Space Robots-Basic Equations and Applications,” 2006 IEEE/RSJ International Conference on Intelligent Robots and Systems, Beijing, 9-15 October 2006, pp. 3137-3142.

[6] T. Malik and S. Writer, "Orbital Express: Prototype Satellites Primed for In-Flight Service,” 2013.

http://www.space.com/3546-orbital-express-prototype-sat ellites-primed-flight-service.html

[7] P. K. Wettergreen, L. Pedersen, D. Kortenkamp, D. Wettergreen and I. Nourbakhsh, "A Survey of Space Robotics," 2003.

[8] ESA, "Rosetta at a Glance,” European Space Agency, 2013.

http://www.esa.int/Our_Activities/Space_Science/Rosetta /Rosetta_at_a_glance2

[9] W. Bluethmann, R. Ambrose, M. Diftler, S. Askew, E. Huber, M. Goza, F. Rehnmark, C. Lovchik and D. Magruder, "Robonaut: A Robot Designed to Work with Humans in Space," Autonomous Robots, Vol. 14, No. 2-3, 2003, pp. 179-197. doi:10.1023/A:1022231703061

[10] M. A. Diftler, T. D. Ahlstrom, R. O. Ambrose, N. A. Radford, C. A. Joyce, N. De La Pena, A. H. Parsons and A. L. Noblitt, "Robonaut 2-Initial Activities On-Board the ISS,” 2012 IEEE Aerospace Conference, Big Sky, 3-10 March 2012, pp. 1-12. doi:10.1109/AERO.2012.6187268

[11] R. Doyle, E. Dupuis, M. Oda, J.-C. Piedbeouf and G. Visentin, "Progress on AI, Robotics, and Automation in Space: A Report from i-SAIRAS 08,” IEEE Intelligent Systems, Vol. 24, No. 1, 2009, pp. 78-83. doi:10.1109/MIS.2009.16

[12] "National Geographic Channel, Living on the Moon Clip 3,” 2013.

http://www.youtube.com/watch?v=jhJgR6QhNPU\&featu re=youtube_gdata_player

[13] ESA, “Eurobot Ground Prototype,” European Space Agency, 2013.

http://www.esa.int/Our_Activities/Human_Spaceflight/Re search/Eurobot_Ground_Prototype

[14] J. Borland, "Mars Rover Dips, Slips into Tricky Territory,” Wired Science, 2007.

http://www.wired.com/wiredscience/2007/09/mars-roverdips/

[15] NASA, “Mars Exploration Program: NASA in 2018 ExoMars Rover,” 2013. http://mars.jpl.nasa.gov/programmissions/missions/future/ exomarsrover2018/

[16] “Mars Science Laboratory: Technology,” 2013. http://mars.jpl.nasa.gov/msl/mission/technology/ 
[17] NASA, “Robonaut,” 2013. http://robonaut.jsc.nasa.gov/default.asp

[18] E. T. Baumgartner, R. G. Bonitz, J. P. Melko, L. R. Shiraishi, P. C. Leger and A. Trebi-Ollennu, "Mobile Manipulation for the Mars Exploration Rover-A Dexterous and Robust Instrument Positioning System," IEEE Robotics \& Automation Magazine, Vol. 13, No. 2, 2006, pp. 27-36. doi:10.1109/MRA.2006.1638013

[19] S. Jin, J. Cho, X. D. Pham, K.-M. Lee, S.-K. Park, M. Kim and J. W. Jeon, "FPGA Design and Implementation of a Real-Time Stereo Vision System,” IEEE Transactions on Circuits and Systems for Video Technology, Vol. 20, No. 1, 2010, pp. 15-26.

[20] “FPGA Stereo Vision Project,” 2013. http://danstrother.com/2011/01/24/fpga-stereo-vision-proj ect/

[21] G. Reina, L. Ojeda, A. Milella and J. Borenstein, "Wheel Slippage and Sinkage Detection for Planetary Rovers," IEEE/ASME Transactions on Mechatronics, Vol. 11, No. 2, 2006, pp. 185-195. doi:10.1109/TMECH.2006.871095

[22] G. Reina, A. Milella and F. W. Panella, "Vision-Based
Wheel Sinkage Estimation for Rough-Terrain Mobile Robots," 15th International Conference on Mechatronics and Machine Vision in Practice, Auckland, 2-4 December 2008, pp. 75-80. doi:10.1109/MMVIP.2008.4749510

[23] P.-Y. Cui, F.-Z. Yue and H.-T. Cui, "Stereo Vision Based Motion Estimation for Lunar Rover Navigation,” 2006 International Conference on Machine Learning and Cybernetics, Dalian, 13-16 August 2006, pp. 3847-3852. doi:10.1109/ICMLC.2006.258696

[24] T. Fong, L. M. Hiatt, C. Kunz and M. Bugajska, "The Human-Robot Interaction Operating System,” Proceedings of the 1st ACM SIGCHI/SIGART Conference on Human-Robot Interaction, New York, 2006, pp. 41-48

[25] L. B. Bridgwater, C. A. Ihrke, M. A. Diftler, M. E. Abdallah, N. A. Radford, J. M. Rogers, S. Yayathi, R. S. Askew and D. M. Linn, "The Robonaut 2 Hand-Designed to Do Work with Tools," 2012 IEEE International Conference on Robotics and Automation, Saint Paul, 14-18 May 2012, pp. 3425-3430. doi:10.1109/ICRA.2012.6224772 